\title{
UTILIZAÇÃO DA ANÁLISE DE COMPONENTES INDEPENDENTES PARA ELIMINAR PISCADAS DE SINAIS DE ELETROENCEFALOGRAFIA
}

\author{
A. M. Freitas*, A. N. Silva*, M. R. Azevedo* e A. B. Soares* \\ *Universidade Federal de Uberlândia, Uberlândia, Minas Gerais \\ e-mail: amanda.medeirosf@yahoo.com.br
}

Resumo: A Análise Componentes Independentes é uma ferramenta matemática que pode ser aplicada como filtro de artefatos em sinais biomédicos. Dentro destes artefatos, os sinais oculares entram como um dos principais artefatos para os sinais de Eletroencefalografia. Entretanto, a utilização de diferentes tamanhos de janela do sinal pode interferir consideravelmente no resultado final do filtro. Portanto, o presente estudo visa investigar a influência que o tamanho da janela pode causar nos resultados finais para filtragem de artefatos oculares em sinais de eletroencefalografia.

Palavras-chave: Análise de componentes independentes, eletroencefalografia, artefato de piscada, filtro para piscada.

Abstract: The Independent Component Analysis is a mathematical method that can be applied as some filter artifacts in biomedical signals. Within these artifacts, blink signals are one of the main artifacts for EEG signals. However, the use of different window sizes signal may interfere considerably in the end result of the filter. Therefore, the present study aims to investigate the influence of the size of the window may cause the final results to filter blinks artifacts in EEG signals.

Keywords: Independent Component Analysis, Electroencephalography, blinks artifacts, filter to blinks.

\section{Introdução}

A eletroencefalografia (EEG) é responsável por analisar e estudar o comportamento elétrico das atividades corticais espontâneas, mensuradas por meio de eletrodos de superfície posicionados sobre o escalpo. Os sinais podem ser coletados com o intuito de monitorar o comportamento cortical, diagnosticar alguma disfunção e também para estabelecer interfaces cérebro-computador (ICC), proporcionando vias de comunicação alternativas para pessoas com disfunções motoras severas.

Entretanto, os sinais de EEG são facilmente perturbados por artefatos oculares, como a movimentação e a piscada dos olhos, causando sérios problemas na análise e interpretação dos sinais coletados. Por exemplo, a amplitude (tensão) resultante de uma piscada de olhos pode ser 10 vezes maior do que a amplitude do sinal cortical normal [1], o que pode prejudicar o desempenho e a acurácia das ICCs, principalmente aquelas que utilizam potenciais evocados visuais [2].

Para solucionar este problema de piscada de olhos, alguns estudos orientam que, durante a apresentação do estímulo, o indivíduo não deve fechar os olhos [3]-[5]. Outra estratégia comum é a eliminação das épocas que contém amplitude maiores que um limiar estabelecido, geralmente, $50 \mu \mathrm{V}$ [6]-[8]. Entretanto, quando os estímulos e as épocas são limitados, ou quando os artefatos ocorrem com frequência, a quantidade de dados perdida em decorrência desta estratégica pode se tornar inviável. Conforme exposto por Jung et al., um experimento conduzido com crianças autistas pode apresentar artefatos oculares em praticamente $100 \%$ das épocas [6]. Alguns grupos de pacientes também podem apresentar resultados similares, inviabilizando praticamente todos os dados coletados.

Com esta problemática, nos últimos anos muitos estudos focaram em propostas para eliminar artefatos oculares presentes nos sinais de EEG. Dentre estas propostas, a análise de componentes independentes (ACI) recebeu muita ênfase devido ao seu potencial no processamento de sinais biomédicos, podendo ser utilizada como filtro dos sinais corticais em decorrência da identificação das componentes independentes [2]. Entretanto, quando alguns parâmetros da ACI são alterados, como o tamanho da janela do sinal a ser filtrado, os resultados da filtragem mudam consideravelmente.

Portanto, o presente estudo visa analisar a utilização da ACI como ferramenta de filtro para eliminar os artefatos de piscadas dos sinais de EEG, objetivando investigar a influência que o tamanho da janela pode causar nos resultados finais da filtragem.

A ACI é uma técnica matemática que utiliza o princípio da separação de fontes cegas, visando a recuperação de sinais independentes que são misturados linearmente de forma desconhecida, sendo observados nos sensores de coleta. Esta técnica reduz a dependência estatística das componentes, tentando tornar os sinais os mais independentes possíveis [9].

Uma vez que as componentes independentes (CIs) foram encontradas, é possível filtrar aquela componente que mais se assemelha ao sinal de piscada dos olhos, retirando assim estes artefatos dos sinais corticais. Entretanto, encontrar as componentes que mais se 
assemelham à piscada não é uma tarefa trivial, nem sempre as componentes apresentam correlação satisfatória com o sinal que se pretende eliminar. Se não for encontrada nenhuma componente principal que mostre uma correlação satisfatória com o sinal que deseja eliminar, a época precisa ser eliminada.

\section{Materiais e métodos}

O presente estudo faz parte de um amplo projeto de pesquisa que está em desenvolvimento no Laboratório de Engenharia Biomédica (BIOLAB) da Universidade Federal de Uberlândia (UFU). Portanto, os dados a serem expostos aqui foram adquiridos no banco de dados do projeto relatado, o qual possui aprovação do Comitê de Ética em Pesquisa da UFU por meio do número 650.649 .

Deste banco de dados, foi escolhido os sinais de um voluntário aleatório para compor a análise da presente pesquisa. Conforme metodologia estabelecida no projeto, o voluntário foi submetido a 60 estímulos visuais com duração de 0,5 segundos e intervalo entre estímulos de 3 segundos. Os estímulos visuais eram compostos por imagens que geravam reações positivas, negativas e neutras, de acordo com suas valências estabelecidas pelo International Affective Picture System (IAPS) [10]. Estas imagens com diferentes valências eram apresentadas de forma aleatória ao voluntário, seguindo a premissa de que uma imagem não podia ser apresentada mais de uma vez.

Durante o experimento, não foi dado nenhuma orientação em relação às piscadas dos olhos, portanto, as piscadas eram realizadas de forma aleatória, de acordo com a necessidade do voluntário.

Antes de realizar o processamento utilizando a ACI, foi realizado um pré-processamento para filtrar as bandas de frequência que não pertence ao espectro característicos dos sinais corticais. Portanto, foi implementado um filtro Butterworth passa faixa entre $0.1 \mathrm{~Hz}$ à $50 \mathrm{~Hz}$, e também um filtro rejeita faixa de 60 Hz. Este filtro rejeita faixa foi utilizado devido à taxa de atenuação do filtro Butterworth, que possui características lentas para números de pólos pequenos.

Para analisar o efeito do tempo na filtragem utilizando a ACI, foram consideradas 3 janelas com tamanhos diferentes: 1 segundo antes do estímulo até 1 segundo depois do início do estímulo; a duração entre a piscada anterior e a piscada posterior em relação à uma piscada que estava dentro do intervalo do estímulo; e a duração máxima de 3 piscadas inteiras, ou seja, não poderia conter a metade de uma onda relacionada à piscada. Estes três tempos diferentes podem ser observados nas Figuras 1 (a), (b) e (c), respectivamente.

Uma vez que estas janelas foram processadas com a ACI, foi necessário estabelecer qual das componentes principais resultantes era a mais próxima da onda original de piscadas dos olhos. Para isto, foi feito a correlação entre o sinal do canal de EEG Fpl com todas as componentes principais. A componente que apresentasse o valor de correlação mais alto, era zerada.
Assim, o sinal original de EEG era expresso sem a componente de piscada, mantendo apenas os sinais corticais.

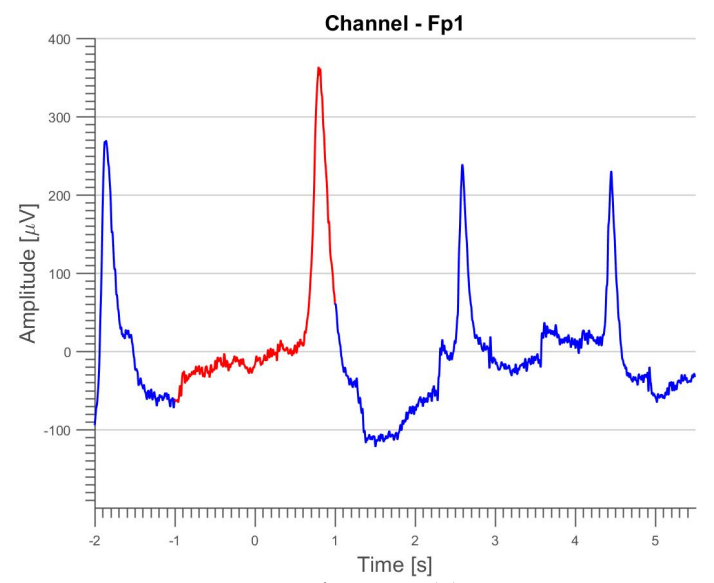

Figura 1 (a)

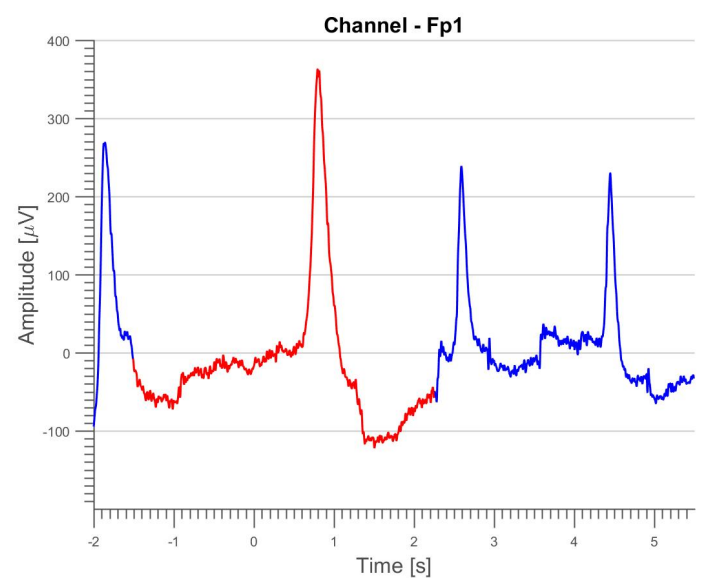

Figura 1 (b)

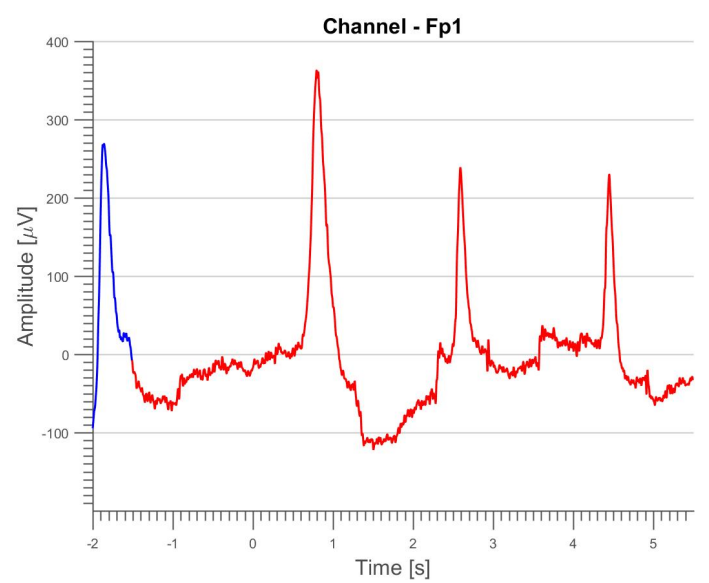

Figura 1 (c)

Figura 1 - Janelas dos sinais de EEG com diferentes tempos de duração para análise, sendo (a) com duração de 2 segundos, 1 segundo antes do estímulo e 1 segundo depois do início do estímulo; (b) intervalo entre uma piscada anterior e uma piscada posterior à piscada que está dentro do intervalo de estímulo; (c) máxima duração entre 3 piscadas completas. 


\section{Resultados}

Para cada uma das três janelas selecionadas para implementação da ACI, foi realizada a correlação das componentes independentes com o canal de EEG Fp1. Os resultados da melhor correlação entre as CIs e Fp1, para cada tamanho de janela estão apresentados na Tabela 1.

Tabela 1- Correlação resultante entre as componentes independentes e o sinal do canal Fp1 do EEG para cada tamanho de janela, conforme a metodologia estabelecida

\begin{tabular}{lccc}
\hline & Janela 1 & Janela 2 & Janela 3 \\
\hline $\begin{array}{l}\text { Correlação } \\
\text { resultante }\end{array}$ & $9,88 \%$ & $66,55 \%$ & $84,85 \%$ \\
\hline
\end{tabular}

$\mathrm{Na}$ literatura é comum encontrar artigos que utilizam apenas as componentes independentes que obtiveram correlação superior à $80 \%$ com sinal que deseja eliminar [1], [2], [6], [9]. Portanto, apenas a Janela 3, com duração máxima de 3 piscadas, apresentou correlação satisfatória para implementar o filtro. As janelas 1 e 2 não apresentaram desempenho suficiente para que as componentes fossem filtradas.

$\mathrm{O}$ sinal original de Fp1 e o sinal filtrado utilizando ICA com a componente que apresentou $84,85 \%$ de correlação, estão expostos na Figura 2 (a) e 2 (b).

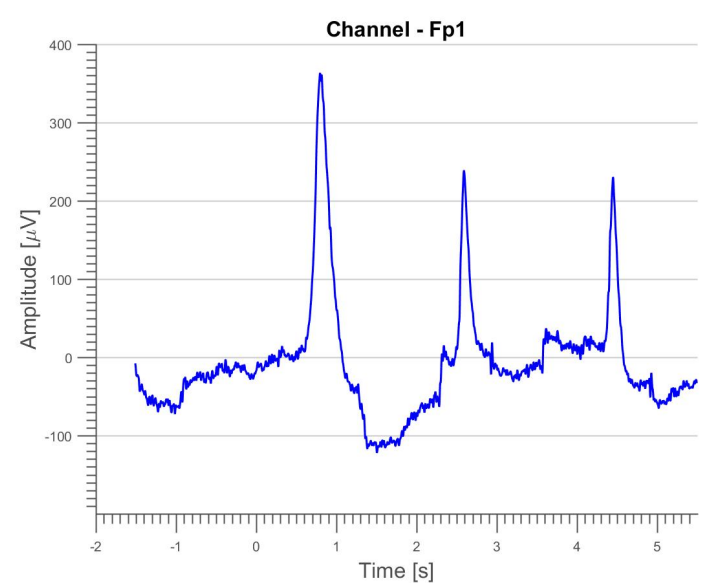

Figura 2 (a)

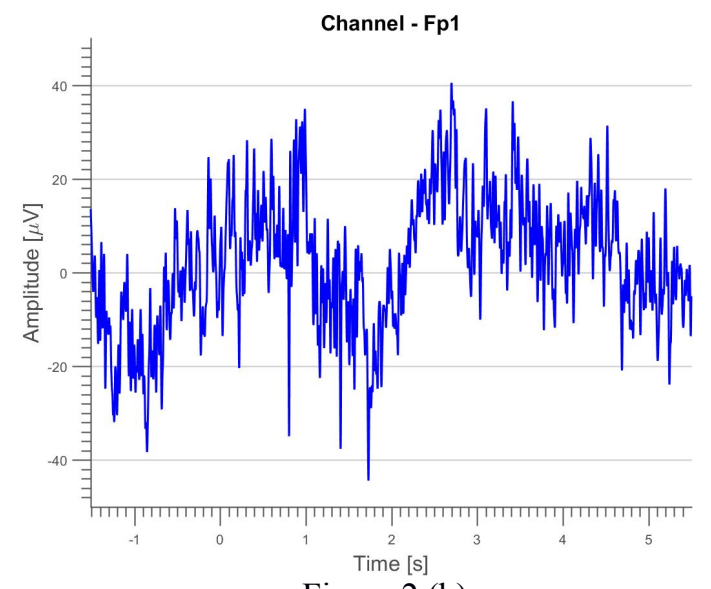

Figura 2 (b)

Figura 2 - Resultado final do sinal de Fp1 filtrado utilizando ICA e a componente principal com maior correlação. Em (a) observa-se o sinal original e em (b) o sinal filtrado

\section{Discussão}

Com os resultados obtidos foi possível observar que o tamanho da janela do sinal analisado pela ACI interfere na detecção das componentes independentes. Conforme mostra a Tabela 1, foi observado que, quanto maior for a janela, maior é a correlação entre a componente independente e o sinal do artefato. Entretanto, em análise prévias, foi possível identificar que, se a janela a ser analisada possuir uma piscada incompleta, sem início ou fim, as componentes resultantes não conseguem reproduzir este sinal.

Provavelmente, esta variação da correlação das componentes independentes em relação ao tempo da janela e também à forma da onda da piscada (onda completa ou incompleta) ocorre porque o cálculo matemático não consegue garantir que aquele sinal representa um comportamento aleatório e independente de todas as componentes do sinal, o que é o pressuposto básico para aplicar a ACI.

Portanto, quando for aplicar a ACI com o objetivo de filtrar artefatos oculares, é necessário garantir que a janela do sinal tenha a duração máxima possível sem que haja piscadas incompletas. Para garantir isto, uma possível implementação é encontrar os picos do sinal (piscadas) e determinar o tempo em que começa e termina a picada, podendo ser estabelecido pela duração média da piscada, $200 \mathrm{~ms}$ [11], [12], ou por meio da média e do desvio padrão (z-score).

Entretanto, esta duração máxima possível não pode ser demasiadamente grande, pois, se as formas de onda das picadas começarem a diferirem entre si, as piscadas serão representadas por CIs diferentes, ao invés de apenas uma CI conter todas as piscadas. Por exemplo, para o mesmo sinal, a janela de análise foi aumentada para 10 segundos, o que resultou em uma correlação de $79,4 \%$ com o canal Fp1. Já para uma janela com 60 segundos, a correlação foi de apenas $65,12 \%$, o que 
tornaria inviável para filtragem desta componente independente.

O sinal filtrado exposto na Figura 2 (b) mostra que o artefato de piscada foi eliminado de forma satisfatória. $\mathrm{O}$ sinal cortical filtrado apresentou as mesmas características de sua onda original, entretanto não é possível visualizar as ondas referentes às piscadas. Este resultado mostra que, para este tempo de aproximadamente 6 segundos e com 3 ondas completas de piscada, a filtragem apresentou bons resultados.

\section{Conclusão}

Com os resultados do presente estudo, é possível concluir que a utilização da ACI é satisfatória para eliminar artefatos de piscadas de olhos. Entretanto, é importante ressaltar que a duração da janela a ser analisada interfere consideravelmente no resultado final das componentes independentes.

\section{Agradecimentos}

Agradecimento aos órgãos de fomento: Conselho Nacional de Desenvolvimento Científico e Tecnológico (CNPq) e a Coordenação de Aperfeiçoamento de Pessoal de Nível Superior (Capes). Ao laboratório de Engenharia Biomédica da Faculdade de Engenharia Elétrica - UFU.

\section{Referências}

[1] C. A. Joyce, I. F. Gorodnitsky, and M. Kutas. Automatic removal of eye movement and blink artifacts from EEG data using blind component separation. Psychophysiology, vol. 41, no. 2, pp. 313-325, 2004.

[2] Zhaojun Xue, Jia Li, Song Li, and Baikun Wan, Using ICA to Remove Eye Blink and Power Line Artifacts in EEG. First International Conference on Innovative Computing, Information and Control - Volume I (ICICIC'06), 2006, vol. 3, pp. 107-110.

[3] J. Höhne and M. Tangermann. Towards UserFriendly Spelling with an Auditory BrainComputer Interface: The CharStreamer Paradigm. PLoS One, vol. 9, no. 6, p. e98322, Jun. 2014.

[4] I. Käthner, C. A. Ruf, E. Pasqualotto, C. Braun, N. Birbaumer, and S. Halder. A portable auditory P300 brain-computer interface with directional cues. Clin. Neurophysiol. vol. 124, no. 2, pp. 327-338, Feb. 2013.

[5] H. Ramoser, J. Muller-Gerking, and G. Pfurtscheller. Optimal spatial filtering of single trial EEG during imagined hand movement. IEEE Trans. Rehabil. Eng. vol. 8, no. 4, pp. 441-446, 2000.
[6] T.-P. Jung, S. Makeig, M. Westerfield, J. Townsend, E. Courchesne, and T. J. Sejnowski. Removal of eye activity artifacts from visual event-related potentials in normal and clinical subjects. Clin. Neurophysiol. vol. 111, no. 10, pp. $1745-1758$, Oct. 2000.

[7] M. Guo, G. Xu, L. Wang, M. Masters, G. Milsap, N. Thakor, and A. B. Soares. The anterior contralateral response improves performance in a single trial auditory oddball BMI. Biomed. Signal Process. Control. vol. 22, pp. 74-84, Sep. 2015.

[8] D. Moretti, F. Babiloni, F. Carducci, F. Cincotti, E. Remondini, P. Rossini, S. Salinari, and C. Babiloni. Computerized processing of EEGEOG-EMG artifacts for multi-centric studies in EEG oscillations and event-related potentials. Int. J. Psychophysiol. vol. 47, no. 3, pp. 199216, 2003.

[9] A. Hyvärinen and E. Oja. Independent component analysis: algorithms and applications. Neural Networks. vol. 13, no. 4, pp. 411-430, 2000.

[10] P. J. Lang, M. M. Bradley, and B. N. Cuthbert. International affective picture system (IAPS): Affective ratings of pictures and instruction manual. Tech. Rep. A-8. Univ. Florida, Gainesville, FL, 2008.

[11] K. A. Glass, G. A. Frishkoff, R. M. Frank, C. Davey, J. Dien, A. D. Malony, and D. M. Tucker. A Framework for Evaluating ICA Methods of Artifact Removal from Multichannel EEG. Springer Berlin Heidelberg, 2004, pp. 1033-1040.

[12] G. Barbato, G. Ficca, G. Muscettola, M. Fichele, M. Beatrice, and F. Rinaldi. Diurnal variation in spontaneous eye-blink rate. Psychiatry Res. vol. 93, no. 2, pp. 145-151, 2000. 